\title{
Is Weibull Distribution the Most Appropriate Statistical Strength Distribution FOR BRitTle MATERIAls?
}

\author{
Bikramjit Basu ${ }^{1}$, Devesh Tiwari $^{2}$, Debasis Kundu ${ }^{3}$ and Rajesh Prasad ${ }^{1}$
}

\begin{abstract}
Strength reliability, one of the critical factors restricting wider use of brittle materials in various structural applications, is commonly characterized by Weibull strength distribution function. In the present work, the detailed statistical analysis of the strength data is carried out using a larger class of probability models including Weibull, normal, log-normal, gamma and generalized exponential distributions. Our analysis is validated using the strength data, measured with a number of structural ceramic materials and a glass material. An important implication of the present study is that the gamma or log-normal distribution function, in contrast to Weibull distribution, may describe more appropriately, in certain cases, the experimentally measured strength data.
\end{abstract}

Key Words And Phrases: Two-parameter distributions, Kolmogorov Statistics, $\chi^{2}$ statistics, shape parameter, scale parameter, strength data.

${ }^{1}$ Department of Materials and Metallurgical Engineering, Indian Institute of Technology Kanpur, Pin 208016, India.

${ }^{2}$ Department of Computer Science and Engineering, Indian Institute of Technology Kanpur, Pin 208016, India.

${ }^{3}$ Department of Mathematics and Statistics, Indian Institute of Technology Kanpur, Pin 208016, India. 


\section{Introduction}

Brittle materials, like ceramics have many useful properties like high hardness, stiffness and elastic modulus, wear resistance, high strength retention at elevated temperatures, corrosion resistance associated with chemical inertness etc $^{1}$. The advancement of ceramic science in the last few decades has enabled the application of this class of materials to evolve from more traditional applications (sanitary wares, pottery etc.) to cutting edge technologies, including rocket engine nozzles, engine parts, implant materials for biomedical applications, heat resistant tiles for space shuttle, nuclear materials, storage and renewable energy devices, fiber optics for high speed communications and elements for integrated electronics like MicroElectro-Mechanical Systems (MEMS).

In many of the engineering applications requiring load bearing capability i.e. structural applications, it has been realized over the years that an optimum combination of high toughness with high hardness and strength reliability is required ${ }^{2}$. Despite having much better hardness compared to conventional metallic materials, the major limitations of ceramics for structural and specific non-structural applications are the poor toughness and low strength reliability ${ }^{3}$. The poor reliability in strength or rather large variability in strength property of ceramics is largely due to the variability in distribution of crack size, shape and orientation with respect to the tensile loading axis ${ }^{4}$. Consequently, the strength of identical ceramic specimens under identical loading conditions is different for a given ceramic material. The physics of the fracture of brittle solids and the origin of strength theory is discussed in some details in section 2 .

The above mentioned limitations have triggered extensive research activities in the ceramic community to explore several toughening mechanisms ${ }^{5}$, and to adopt refined processing routes $^{6}$ in order to develop tough ceramics with reliable strength. The major focus of the 
present work is however the strength characterization of brittle materials.

In a recent paper ${ }^{7}, \mathrm{Lu}$ et al. analyzed the fracture statistics of brittle materials using Weibull and normal distributions. They have considered the strength data of three different ceramic materials, i.e. silicon nitride $\left(\mathrm{Si}_{3} \mathrm{~N}_{4}\right)$, silicon carbide $(\mathrm{SiC})$ and zinc oxide $(\mathrm{ZnO})$. They used three-parameter Weibull, two-parameter Weibull and normal distributions to analyze these data. It is observed that based on the Akaike Information Criterion (AIC), two-parameter Weibull or normal distributions fit better than the three-parameter Weibull distribution. Although two-parameter Weibull distribution has been widely used in practice to model strength data, Lu et al. ${ }^{7}$ questioned the uncritical use of Weibull distribution in general.

In the present work, we analyze the strength data, obtained in our previous work on monolithic $\mathrm{ZrO}_{2}$ and $\mathrm{ZrO}_{2}-\mathrm{TiB}_{2}$ composites. Additionally, two more strength datasets, one for glass (unknown composition) and other for $\mathrm{Si}_{3} \mathrm{~N}_{4}$ ceramics are selected from available literature. Such a selection of strength dataset will allow us to statistically analyze the strength property of a range of materials i.e. extremely brittle solid like glass to relatively tougher engineering ceramics, like $\mathrm{Si}_{3} \mathrm{~N}_{4} / \mathrm{ZrO}_{2}$ - based materials. In our analysis, a much larger class of probabilistic models has been used. It is to be noted that the strength is always positive and therefore, it is reasonable to analyze the strength data using the probability distribution, which has support only on the positive real axis. Based on this simple idea we have attempted different two-parameter distributions namely, Weibull, gamma, log-normal and generalized exponential distributions. It should be mentioned here that all the above distributions have shape and scale parameters. As the name suggests the shape parameter of each distribution governs the shape of the respective density and distribution functions. For comparison purposes, we have also fitted normal distribution to both datasets, although it does not have the shape parameter and it has the support on the whole real line. 


\section{Physics of the FRACTURE OF BRITTLE SOLIDS}

The variability in strength of ceramics is primarily due to the extreme sensitivity of the presence of cracks of different sizes. It can be noted that the Yield strength and the fracture/failure strength of polycrystalline metals is deterministic and is volume independent, when the characteristic micro-structural feature (grain size) remained the same for the tested metallic samples. However, the fracture strength of a brittle material is, in particular, determined by the critical crack length according to the Griffith's theory ${ }^{8}$ :

$$
\sigma_{f}=\frac{K_{I C}}{\sqrt{\pi a}}
$$

where $\sigma_{f}$ the failure or fracture strength, $K_{I C}$, the critical stress intensity factor (a measure of fracture toughness) under mode-I (tensile) loading and ' $a$ ' the half of the critical or largest crack size.

For a given ceramic material the distribution of crack size, shape, and orientation differs from sample to sample. It is experimentally reported that the strength of ceramics varies unpredictably even if identical specimens are tested under identical loading conditions ${ }^{4}$. In particular, the mean strength, as determined from a multiplicity of similar tests depends on volume of material stressed, shape of test specimen and nature of loading. It is recognized that strength property needs to be analyzed using different probabilistic approaches, largely because of the fact that the probability of failure or fracture of a given ceramic sample critically depends on the presence of a potentially dangerous crack of size greater than a characteristic critical crack size ${ }^{4}$. Clearly, the probability of finding critical crack size is higher in larger volume test specimens and consequently, the brittle materials do not have any deterministic strength property. Since brittle materials exhibit volume dependent strength behavior, the mean strength decreases as the specimen volume increases. From the initial experimental observations, it was evident that a definite relationship should exist between the 
probability that a specimen will fracture and the stress to which it is subjected. Based on the above observations/ predictions, Weibull ${ }^{9}$ proposed a two parameter distribution function to characterize the strength of brittle materials. The generalized strength distribution law has the following expression: $F(\sigma)=1-e^{-\frac{V}{V_{0}} g(\sigma)}$, where $F(\sigma)$ is the probability of failure at a given stress level ' $\sigma$ ', $V$ is the volume of the material tested, $V_{0}$ is the reference volume and $g(\sigma)$ is the Weibull strength distribution function: $g(\sigma)=\left(\frac{\sigma}{\sigma_{0}}\right)^{m}$, where $m$ is the Weibull modulus and $\sigma_{0}$ is the reference strength for a given reference volume $V_{0}$. The characteristic strength distribution parameter, $m$, indicates the nature, severity and dispersion of flaws ${ }^{2}$. More clearly, a low $m$ value indicates non-uniform distribution of highly variable crack length (broad strength distribution), while a high $m$ value implicates uniform distribution of highly homogeneous flaws with narrower strength distribution. Typically, for structural ceramics, $m$ varies between 3 and 12, depending on the processing conditions ${ }^{1}$. The Weibull distribution function, till to-date, is widely used to model or characterize the fracture strength of various brittle materials like $\mathrm{Al}_{2} \mathrm{O}_{3}, \mathrm{Si}_{3} \mathrm{~N}_{4}$ etc ${ }^{2,10,11}$.

\section{EXPERIMENTS}

As part of the present study, the analysis of four strength datasets is performed. The first two datasets i.e. dataset 1 and dataset 2 are the results of our previous experimental work. In particular, dataset 1 refers to the strength data obtained with hot pressed $\mathrm{ZrO}_{2}$ (2.5 mol \% yttria-stabilized) - 30 vol \% $\mathrm{TiB}_{2}\left(\mathrm{TZP}-\mathrm{TiB}_{2}\right)$ composites; while dataset 2 is obtained during the strength measurement of hot pressed $2 \mathrm{~mol} \%$ yttria-stabilised tetragonal Zirconia (2Y - TZP) monolithic ceramic. Both the selected materials are fully dense (> 97\% theoretical density). The details of the processing, micro-structural characterization as well mechanical properties can be found elsewhere ${ }^{12-15}$. The selection of these particular grades of $\mathrm{ZrO}_{2}$ materials is primarily because of the fact that our recent research in optimizing 
the toughness of TZP-based materials revealed that both the selected 2Y-TZP monoliths and the TZP-TiB ${ }_{2}$ composite exhibited best fracture toughness (2Y-TZP: $10.2 \pm 0.4 \mathrm{MPa}$ $\mathrm{m}^{1 / 2} ; \mathrm{TZP} \mathrm{TiB}_{2}: 10.3 \pm 0.5 \mathrm{MPa} \mathrm{m}^{1 / 2}$ ) of all the developed materials ${ }^{13-15}$. Therefore, detailed tribiological characterization as well as strength measurement was carried out on these optimized materials ${ }^{14}$. The micro-structural characterization study using SEM and TEM revealed the homogeneous distribution of coarser $\mathrm{TiB}_{2}$ particles (average size $\sim 1 \mu \mathrm{m}$ ) in TZP matrix. The average $\mathrm{ZrO}_{2}$ grain size in both monolith and composite is $\sim 0.3-0.4 \mu \mathrm{m}$. Because of the use of highly pure commercial starting powders, the presence of any grain boundary crystalline/amorphous phase neither in monolith nor in composite was detected using high resolution TEM study ${ }^{15}$.

The flexural strength of both $\mathrm{ZrO}_{2}$ monolith and composite at room temperature was measured using a 3-point bending test configuration. The test specimens with typical dimension of $25.0 \times 5.4 \times 2.1 \mathrm{~mm}$, were machined out of the hot pressed disks. The span width was $20 \mathrm{~mm}$ with a cross head speed of $0.1 \mathrm{~mm} / \mathrm{min}$. At least 15 identical specimens were tested for each material grade. The fracture surface observations using SEM predominantly indicated intergranular fracture in both $\mathrm{ZrO}_{2}$ monolith and composites. Also detailed microscopy study indicated similarity in fracture origin for both the selected materials i.e. the critical surface flaw, located on the tensile face of the bend specimen.

Among the four selected datasets, the other two datasets are taken from literature. While dataset 3 is obtained using sintered $\mathrm{Si}_{3} \mathrm{~N}_{4}$ materials ${ }^{16}$, the dataset 4 is reported to be recorded from the brittle glass of unknown composition ${ }^{17}$. It can be mentioned here that $\mathrm{Si}_{3} \mathrm{~N}_{4}$-based materials have been widely researched in the ceramics community for their potential high temperature applications, like engine components etc. The details of the strength measurements and microstructural details of the selected $\mathrm{Si}_{3} \mathrm{~N}_{4}$ materials can be found elsewhere ${ }^{16}$. In reference [17], the 3-point flexural strength measurement is reported for an unknown glass 
compositions. Typical bend bar dimension of glass sample was $3 \times 4 \times 40 \mathrm{~mm}$ with span length of $30 \mathrm{~mm}$. The crosshead velocity was $0.5 \mathrm{~mm} / \mathrm{min}$.

\section{Different Competing Models}

In this section we briefly describe different competing probabilistic models considered here and mention the estimation procedures of the unknown parameters from a given sample dataset $\left\{x_{1}, \ldots, x_{n}\right\}$.

\subsection{Weibull Distribution}

The density function of the two-parameter Weibull distribution for $\alpha>0$ and $\lambda>0$ has the following form:

$$
f_{W E}(x ; \alpha, \lambda)=\alpha \lambda^{\alpha} x^{\alpha-1} e^{-(\lambda x)^{\alpha}} .
$$

Here $\alpha$ and $\lambda$ represent the shape and scale parameters respectively. Therefore, the maximum likelihood estimators of $\alpha$ and $\lambda$ can be obtained by maximizing the following log-likelihood function with respect to the unknown parameters;

$$
L_{W E}\left(\alpha, \lambda \mid x_{1}, \ldots, x_{n}\right)=n \ln \alpha+(n \alpha) \ln \lambda+(\alpha-1) \sum_{i=1}^{n} \ln x_{i}-\lambda^{\alpha} \sum_{i=1}^{n} x_{i}^{\alpha} .
$$

Note that if $(\hat{\alpha}, \hat{\lambda})$ maximize $(2)$ then

$$
\hat{\lambda}=\left(\frac{n}{\sum_{i=1}^{n} x_{i}^{\hat{\alpha}}}\right)^{\frac{1}{\hat{\alpha}}}
$$

and $\hat{\alpha}$ can be obtained by maximizing the profile log-likelihood of $\alpha$ as given below;

$$
P_{W E}(\alpha)=n \ln \alpha-n \ln \left(\sum_{i=1}^{n} x_{i}^{\alpha}\right)+(\alpha-1) \sum_{i=1}^{n} \ln x_{i} .
$$

Since (4) is a unimodal function, the maximization of $P_{W E}(\alpha)$ is not a difficult problem. 


\subsection{Gamma Distribution}

The two-parameter gamma distribution for $\alpha>0$ and $\lambda>0$ has the following density function;

$$
f_{G A}(x ; \alpha, \lambda)=\frac{\lambda^{\alpha}}{\Gamma(\alpha)} x^{\alpha-1} e^{-\lambda x} .
$$

Here also $\alpha, \lambda$ represent the shape and scale parameters respectively and $\Gamma(\alpha)$ is the incomplete gamma function defined by

$$
\Gamma(\alpha)=\int_{0}^{\infty} x^{\alpha-1} e^{-x} d x
$$

The maximum likelihood estimators of $\alpha$ and $\lambda$ can be obtained by maximizing the loglikelihood function

$$
L_{G A}\left(\alpha, \lambda \mid x_{1}, \ldots, x_{n}\right)=n \alpha \ln \lambda-n \ln (\Gamma(\lambda))+(\alpha-1) \sum_{i=1}^{n} \ln x_{i}-\lambda \sum_{i=1}^{n} x_{i} .
$$

with respect to the unknown parameters. Therefore, if $\hat{\alpha}$ and $\hat{\lambda}$ are the maximum likelihood estimators of $\alpha$ and $\lambda$ respectively, then

$$
\hat{\lambda}=\frac{\hat{\alpha}}{\frac{1}{n} \sum_{i=1}^{n} x_{i}},
$$

moreover, the maximum likelihood estimator of $\alpha$ can be obtained by maximizing

$$
P_{G A}(\alpha)=\alpha n(\ln \alpha-1)-n \ln (\Gamma(\alpha))+\alpha \sum_{i=1}^{n} \ln x_{i}
$$

\subsection{Log-Normal Distribution}

The density function of the two-parameter log-normal distribution with scale parameter $\lambda$ and shape parameter $\alpha$ is as follows;

$$
f_{L N}(x ; \alpha, \lambda)=\frac{1}{\sqrt{2 \pi} x \alpha} e^{-\left[(\ln x-\ln \lambda)^{2} / 2 \alpha^{2}\right]} .
$$


The maximum likelihood estimators of the unknown parameters can be obtained by maximizing the log-likelihood function of the observed data

$$
L_{L N}\left(\alpha, \lambda \mid x_{1}, \ldots, x_{n}\right)=-\sum_{i=1}^{n} \ln x_{i}-n \ln \alpha-\sum_{i=1}^{n} \frac{(\ln x-\ln \lambda)^{2}}{\alpha^{2}} .
$$

Interestingly, unlike Weibull or gamma distributions, the maximum likelihood estimators of $\alpha$ and $\lambda$ can be obtained explicitly and they are as follows;

$$
\hat{\lambda}=\left(\prod_{i=1}^{n} x_{i}\right)^{\frac{1}{n}} \quad \text { and } \quad \hat{\alpha}=\left[\frac{1}{n} \sum_{i=1}^{n}\left(\ln x_{i}-\ln \hat{\lambda}\right)^{2}\right]^{\frac{1}{2}} .
$$

\subsection{Generalized Exponential Distribution}

The two-parameter generalized exponential distribution has the density function

$$
f_{G E}(x ; \alpha, \lambda)=\alpha \lambda e^{-\lambda x}\left(1-e^{-\lambda x}\right)^{\alpha-1}
$$

Here $\alpha>0$ and $\lambda>0$ are the shape and scale parameters respectively. Based on the observed data, the log-likelihood function can be written as

$$
L_{G E}\left(\alpha, \lambda \mid x_{1}, \ldots, x_{n}\right)=n \ln \alpha+n \ln \lambda--\lambda \sum_{i=1}^{n} x_{i}+(\alpha-1) \sum_{i=1}^{n} \ln \left(1-e^{-\lambda x_{i}}\right) .
$$

Therefore, $\hat{\alpha}$, the maximum likelihood estimator of $\alpha$, can be written as

$$
\hat{\alpha}=-\frac{n}{\sum_{i=1}^{n} \ln \left(1-e^{-\lambda x_{i}}\right)},
$$

and the maximum likelihood estimator of $\lambda$ can be obtained by maximizing the following profile log-likelihood of $\lambda$,

$$
P_{G E}(\lambda)=-n \ln \left(\sum_{i=1}^{n} \ln \left(1-e^{-\lambda x_{i}}\right)\right)+n \ln \lambda-\lambda \sum_{i=1}^{n} x_{i}-\sum_{i=1}^{n} \ln \left(1-e^{-\lambda x_{i}}\right) .
$$




\section{Different Discrimination Procedures}

In this section we describe different available methods for choosing the best fitted model to a given dataset. For notational simplicity it is assumed that we have only two different classes, but the method can be easily understood for arbitrary number of classes also. Suppose there are two families, say, $\mathcal{F}=\left\{f(x ; \theta) ; \theta \in \mathcal{R}^{p}\right\}$ and $\mathcal{G}=\left\{g(x ; \gamma) ; \gamma \in \mathcal{R}^{q}\right\}$, the problem is to choose the correct family for a given dataset $\left\{x_{1}, \ldots, x_{n}\right\}$. The following methods can be used for model discrimination.

\subsection{Maximum Likelihood CRiterion}

$\mathrm{Cox}^{18}$ proposes to choose the model which yields the largest likelihood function. Therefore, Cox's procedure can be described as follows. Let

$$
T(\hat{\theta}, \hat{\gamma})=\sum_{i=1}^{n} \ln \left(\frac{f\left(x_{i} \mid \hat{\theta}\right)}{g\left(x_{i} \mid \hat{\gamma}\right)}\right)
$$

where $\hat{\theta}$ and $\hat{\gamma}$ are maximum likelihood estimators of $\theta$ and $\gamma$ respectively. Choose the family $\mathcal{F}$ if $T>0$, otherwise choose $\mathcal{G}$. The statistic $T$ is sometimes called the Cox's statistic. It is also observed ${ }^{7}$ that when properly normalized, the statistic $\ln T$ should be asymptotically normally distributed. White ${ }^{19}$ studied the regularity conditions needed for the asymptotic distribution to hold. Marshal et al. ${ }^{20}$ use the likelihood ratio test and by extensive simulation study, they determine the probability of correct selection for different sample sizes. Recently ${ }^{21,22}$, different researchers exploit the asymptotic property of $T$ and determine the minimum sample size which is required for discriminating between different competing models.

In terms of $T$, the above selection procedure is related to a procedure for testing the

hypothesis that the sample came from $\mathcal{F}$ versus that it came from $\mathcal{G}$. This testing problem 
treats the two families asymmetrically and so it is slightly different from the selection problem described above and it is not pursued here.

\subsection{Minimum Distance Criterion}

Among competing models, it is natural to choose a particular model for a given sample, which has the distribution function closest to the empirical distribution function of the data according to some distance measure between the two distribution functions. Note that the empirical distribution function of the given data $\left\{x_{1}, \ldots, x_{n}\right\}$ is given by

$$
F_{n}(x)=\frac{\text { Number of } x_{i} \leq x}{n} \text {. }
$$

The distance between two distribution functions can be defined in several ways, but the most popular distance function between two distribution functions, say $F$ and $G$, is known as the Kolmogorov distance and it can be described as follows;

$$
D(F, G)=\sup _{-\infty<x<\infty}|F(x)-G(x)|
$$

To implement this procedure, a candidate from each parametric family that has the smallest Kolmogorov distance should be found and then the different best fitted distributions should be compared. Unfortunately, the first step of this procedure is difficult both from a theoretical and computational point of view. Practically, from each parametric family the best member

is chosen by maximum likelihood estimators rather than minimizing Kolmogorov distance. Then the family is chosen that provides the best fit to the empirical distribution in the sense of Kolmogorov distance.

\subsection{Minimum Chi-Square Criterion}

This is most probably the oldest method which is being used for goodness of fit or for model discrimination. The basic idea of the minimum chi-square criterion is very simple. First 
divide the sample in $k$ different groups and count the number of observations in each groups. If $f(x, \tilde{\theta})$ and $g(x, \tilde{\gamma})$ are the best fitted models from the families $\mathcal{F}$, and $\mathcal{G}$ respectively, then compute the expected number of observations in each group based on $f(x, \tilde{\theta})$ and $g(x, \tilde{\gamma})$. Suppose the observed frequencies in each group are $n_{1}, \ldots, n_{k}$, and the expected frequencies based on $f(x, \tilde{\theta})$ and $g(x, \tilde{\gamma})$ are $f_{1}, \ldots, f_{k}$ and $g_{1}, \ldots, g_{k}$ respectively, then the chi-square distance between $\left\{x_{1}, \ldots, x_{n}\right\}$ and $f(x, \tilde{\theta})$ is defined as

$$
\chi_{f, \text { data }}^{2}=\sum_{i=1}^{k} \frac{\left(n_{i}-f_{i}\right)^{2}}{f_{i}} .
$$

Similarly, the chi-square distance between $\left\{x_{1}, \ldots, x_{n}\right\}$ and $g(x, \tilde{\gamma})$ is

$$
\chi_{g, \text { data }}^{2}=\sum_{i=1}^{k} \frac{\left(n_{i}-g_{i}\right)^{2}}{g_{i}} .
$$

Now between the two families $\mathcal{F}$ and $\mathcal{G}$ choose family $\mathcal{F}$ if $\chi_{\text {f,data }}^{2}<\chi_{\text {g,data }}^{2}$ and choose family $\mathcal{G}$ otherwise. In this case also, like the previous one, from a given family the best model is chosen using the maximum likelihood estimators. Therefore, $\tilde{\theta}$ and $\tilde{\gamma}$ are chosen as $\hat{\theta}$ and $\hat{\gamma}$ respectively.

\section{Experimental Results}

For datasets 1, 2, 3 and 4, we have fitted different distributions and the estimated parameter values, chi-square values, Kolmogorov distances and the log-likelihood values are reported in Tables 5, 6, 7 and 8 respectively. For datasets 1 to 3, we have divided each data point by 100 and for dataset 4, we subtracted 45 and divided by 10. For each dataset the observed and expected values due to different fitted distributions are also reported in Tables 9, 10, 11 and 12 respectively. We also provide the empirical survival functions and the fitted survival functions for different distributions and for both the datasets in Figures 1, 2, 3 and 4 respectively. 
From Table 5 (see also Figure 1), it is clear that for dataset 1, Weibull is the best fitted model based on the maximum Likelihood criterion or the minimum Kolmogorov distance criterion followed by normal distribution. However, the chi-square value is not the minimum for dataset 1. Since it is well known that the chi-square value may not be that reliable, we accept that for dataset 1, Weibull is the best fitted model among different models considered here. Similar phenomenon is observed for dataset 4. For this set also it is observed that Weibull is the best fitted model in terms of all the criteria (see Table 8 and Figure 4).

The picture is quite different for dataset 2 (see Table 6 and Figure 2) and dataset 3 (see Table 7 and Figure 3). Based on the log-likelihood values, Kolmogorov distance and also the chi-square values, Weibull is the worst fitted model. For dataset 2, apparently gamma and for dataset 3, log-normal are the best fitted models.

Another point that can be mentioned is that the fitted Weibull and normal distributions are closer to each other when compared to the fitted gamma, log-normal and generalized exponential distributions. Therefore, we can make two classes, one with Weibull and normal distributions and the other with gamma, log-normal and generalized exponential distributions. Suppose we take one representative distribution from each group, say Weibull and log-normal. Then based on the result ${ }^{21}$, it is possible to find the probability of correct selection in each case. In fact, the probability of correct selection for datasets 1, 2, 3 and 4 are approximately $78 \%, 82 \%, 77 \%$ and $85 \%$ respectively. Therefore, they are quite high.

For dataset 1, it can be noted that that shape parameter of the Weibull distribution is very high. It shows the symmetric nature of the data whereas dataset 2 is more skewed. Therefore, it is clear that if the strength data are distributed symmetrically around its mean, then Weibull distribution may provide a good fit. However, if it is not, then there may be several good competitors. In our opinion, normal distribution should not be used in fitting strength data, because it may take negative values with high probability. 
In view of the presented statistical analysis as well as that of $\mathrm{Lu}$ et $a l^{7}{ }^{7}$, it is important to revisit the basic theory of Weibull, which links the statistical probability of fracture to the probability of finding a critical crack size in the tested sample. Further investigation should focus on rationalizing/ justifying other strength distribution function from the perspective of the probabilistic theory of brittle fracture.

As a concluding note, the uncritical use of Weibull distribution must be avoided and therefore, the use of Weibull modulus as a strength reliability parameter can only be made after detailed analysis of strength data, as presented in this paper. Similar to the strength data, the grain size parameters, like mean grain size, grain size distribution width are equally im-

portant factors in determining critical material properties. In one of our earlier studies ${ }^{23}$, the use of several statistical distribution functions, like normal, log-normal, Gumbel (Extreme value of type I) was made to evaluate the appropriate distribution function for microstructural description of sintered ceramics, like $\mathrm{ZrO}_{2}$. It was concluded from that study that Gumbel distribution describes much better (statistically) the grain size distribution. However, in many studies, the uncritical use of Gaussian or normal distribution were made to find out grain size distribution parameters for several metals/ ceramic materials. The above discussions evidently places the importance of detailed statistical analysis in evaluating the properties of materials i.e. in a larger scale, in the field of material science.

\section{Conclusions}

In the present work, we have considered several statistical distribution functions with an aim to critically analyze the strength data of brittle materials, like ceramics. Other than Weibull and normal, several two-parameter distributions, like Gamma, Log-normal and generalized exponential distributions were used. The experimentally measured strength data obtained with hot pressed dense ceramics, like monolithic $\mathrm{ZrO}_{2}, \mathrm{ZrO}_{2}-\mathrm{TiB}_{2}$ composites as well as 
literature strength data of $\mathrm{Si}_{3} \mathrm{~N}_{4}$ ceramic and glass were used to validate the statistical analysis. It is observed that the fitted Weibull and normal distributions behave quite similarly, whereas the fitted gamma, log-normal and generalized exponential distributions are of similar nature. Based on the limited set of strength data and using several statistical criteria, like minimum chi-square, minimum Kolmogorov distance and maximum log-likelihood value, the gamma or log-normal distribution function appears to be more appropriate statistical distribution function in some investigated cases. Another important result has been that the probability of correct selection for datasets 1, 2, 3 and 4 are approximately $78 \%, 82 \%, 77 \%$ and $85 \%$ respectively, which are quite high.

The implication of our study is important and that is the strength property of brittle ceramics should be characterized using various statistical criteria and different distribution functions, as adopted in the present work.

\section{ACKNOWLEDGMENT:}

The authors would like to thank Mr. Damodar Tiwari for his constructive suggestions to prepare this manuscript.

\section{References}

[1] Chiang, Y.M., Birnie, D. and Kingery, W.D., Physical Ceramics: Principles for Ceramic Science and Engineering, John Wiley \& Sons Inc., New York, 1997.

[2] Davidge, R.W., Mechanical Behavior of Ceramics, Cambridge University Press, Cambridge, UK, 1979. 
[3] Wachtman, J.B., Mechanical Properties of Ceramics, John Wiley \& Sons, Inc., New York, 1996.

[4] Lawn, B., Fracture of Brittle Solids, Second Edition, Cambridge University Press, Cambridge, UK, 1998.

[5] Basu, B., "Toughening of Y-stabilized tetragonal zirconia ceramics", International Materials Reviews, 2005, 50 [4], 239-256.

[6] Lange, F.F., "Powder processing science and technology for increased reliability", Journal of the American Ceramic Society, 1998, 72, 3 - 15.

[7] Lu, C., Danzer, R. and Fishcer, D. , "Fracture statistics of brittle materials: Weibull or normal distribution", Physical Review E, 2002, 65, 067102.

[8] Griffith, A., Philosophical Transactions of the Royal Society, 1920, A 221, 163.

[9] Weibull, W., "A statistical Distribution Function of wide Applicability", Journal of the Applied Mechanics, 1951, 18, 293 - 305.

[10] Bazant, Z.P., "Probability distribution of energetic-statistical size effect in quasibrittle fracture", Probabilistic Engineering Mechanics, 2004, 19, 307-391.

[11] Danzer, R., Lube, T. and Supancic, P., "Monte Carlo simulations of strength distributions of brittle materials - type of distribution, specimen and sample size", Z. Metallkd, 2001, 92, 773-783.

[12] Basu, B., Zirconia-Titanium Boride Composites for Tribiological Applications, Ph.D. thesis, Katholieke Universiteit Leuven, Belgium, 2001.

[13] Basu, B., Vleugels, J. and Van Der Biest, O., "Toughness tailoring of yttria-doped zirconia ceramics", Material Science Engineering A, 2004, 380, 215-221. 
[14] Basu, B., Vleugels, J. and Van Der Biest, O., "Microstructure-Toughness-Wear relationship of tetragonal zirconia ceramics", Journal of the European Ceramic Society, 2004, 24 [7], 2031-2040.

[15] Basu, B., Vleugels, J. and Van Der Biest, O., "Processing and Mechanical properties of ZrO2-TiB2 composites", Journal of the European Ceramic Society, 2005, (to appear).

[16] Quinn, G.D. and Morrell, R., "Design data for engineering ceramics - a review of the flexure test", Journal of the American Ceramics Society, 1991, 74, 2037 - 2066.

[17] Lu, Y., Cheng, L., Zhang, L., Yan, D. and You, C., "Optimization of sample number for Weibull functions of brittle materials strength", Ceramics International, 2001, 27, 239 241.

[18] Cox, D.R., "Further results on tests of separate families of hypothesis", Journal of the Royal Statistical Society, Ser. B, 1962, 24, 406 - 424.

[19] Marshal, A.W., Meza, J.C. and Olkin, I., "Can data recognize its parent distribution?", Journal of Computational and Graphical Statistics, 2001, 10, 555 - 580.

[20] Kundu, D. and Manglick, A., "Discriminating between the Weibull and Log-normal distributions", Naval Research Logistics, 2004, 51, 893 - 905.

[21] Kundu, D., Gupta, R.D. and Manglick, A., "Discriminating Between the Log-Normal and the Generalized Exponential Distributions", Journal of the Statistical Planning and Inference, 2005, 127, 213 - 227. 
[22] Dierickx, D., Basu, B., Vleugels, J. and Van Der Biest, O., "Statistical extreme value modelling of particle size distributions: experimental grain size distribution type estimation and parameterisation of sintered zirconia", Materials Characterisation, 2000, 45, 61-70. 
Table 1: The experimentally measured flexural strength data (Dataset 1, in MPa units) as obtained with hot pressed $\mathrm{ZrO}_{2}-\mathrm{TiB}_{2}$ composites.

\begin{tabular}{|l|c|c|c|c|c|c|c|c|c|}
\hline Sample No. & 1 & 2 & 3 & 4 & 5 & 6 & 7 & 8 & 9 \\
Value & 495.1 & 628.7 & 1179.4 & 1121.2 & 1028.7 & 871.1 & 1077.0 & 1350.0 & 1320.5 \\
\hline Sample No. & 10 & 11 & 12 & 13 & 14 & 15 & 16 & 17 & 18 \\
Value & 1327.4 & 1070.6 & 1342.7 & 1177.6 & 1226.2 & 1160.5 & 1257.8 & 1214.4 & 1136.5 \\
\hline Sample No. & 19 & 20 & 21 & 22 & & & & & \\
Value & 853.9 & 1084.4 & 1052.8 & 1116.3 & & & & & \\
\hline
\end{tabular}

Table 2: The experimentally measured flexural strength data (Dataset 2, in MPa units) as recorded with hot pressed $\mathrm{ZrO}_{2}$ ceramic.

\begin{tabular}{|l|c|c|c|c|c|c|c|c|c|}
\hline Sample No. & 1 & 2 & 3 & 4 & 5 & 6 & 7 & 8 & 9 \\
Value & 1269.8 & 1290.1 & 1372.3 & 1128.8 & 1243.6 & 1287.6 & 1288.1 & 1381.9 & 995.3 \\
\hline Sample No. & 10 & 11 & 12 & 13 & 14 & 15 & 16 & 17 & 18 \\
Value & 698.5 & 649.8 & 937.1 & 1381.9 & 1228.7 & 1362.5 & 893.5 & 690.3 & 545.0 \\
\hline Sample No. & 19 & 20 & 21 & 22 & 23 & 24 & 25 & 26 & 27 \\
Value & 691.0 & 810.4 & 539.3 & 785.9 & 682.0 & 676.0 & 419.2 & 450.0 & 423.6 \\
\hline Sample No. & 28 & 29 & 30 & 31 & 32 & 33 & & & \\
Value & 488.4 & 353.7 & 619.6 & 631.419 & 648.203 & 527.212 & & & \\
\hline
\end{tabular}

Table 3: The strength data (Dataset 3, in MPa units) of $\mathrm{Si}_{3} \mathrm{~N}_{4}$ ceramic, taken from Ref. 16.

\begin{tabular}{|c|c|c|c|c|c|c|c|c|c|}
\hline Sample No. & 1 & 2 & 3 & 4 & 5 & 6 & 7 & 8 & 9 \\
Value & 373.32 & 421.76 & 421.87 & 450.97 & 464.01 & 511.14 & 517.5 & 512.99 & 556.07 \\
\hline Sample No. & 10 & 11 & 12 & 13 & 14 & 15 & 16 & 17 & 18 \\
Value & 560 & 571.4 & 722.13 & 796.52 & 800.84 & 820.66 & 833.4 & 839.03 & 885.85 \\
\hline
\end{tabular}

Table 4: The strength data (Dataset 4, in MPa units) of glass, taken from Ref. 17.

\begin{tabular}{|c|c|c|c|c|c|c|c|c|c|c|}
\hline Sample No. & 1 & 2 & 3 & 4 & 5 & 6 & 7 & 8 & 9 & 10 \\
Value & 47.7 & 50.2 & 52.4 & 52.5 & 52.9 & 53.8 & 53.9 & 54.6 & 54.7 & 54.9 \\
\hline Sample No. & 11 & 12 & 13 & 14 & 15 & 16 & 17 & 18 & 19 & 20 \\
Value & 55.3 & 55.5 & 56.4 & 57.5 & 59.0 & 60.0 & 61.1 & 61.4 & 62.4 & 62.7 \\
\hline Sample No. & 21 & 22 & 23 & 24 & 25 & 26 & 27 & 28 & 29 & 30 \\
Value & 63.2 & 63.5 & 64.2 & 65.4 & 65.4 & 65.6 & 66.3 & 66.6 & 66.6 & 66.8 \\
\hline Sample No. & 31 & 32 & 33 & 34 & 35 & 36 & 37 & 38 & 39 & 40 \\
Value & 67.2 & 67.5 & 67.6 & 68.0 & 68.4 & 69.6 & 70.4 & 70.7 & 72.6 & 74.4 \\
\hline
\end{tabular}


Table 5: Estimated parameters, K-S distances, log-likelihood values and the fitted chi-square values for different distribution functions of Dataset 1.

\begin{tabular}{|c|c|c|c|c|c|}
\hline Distribution & Shape & Scale & Chi-square & Kolmogorov & Log-likelihood \\
& & & & & \\
Weibull & 7.1033 & 0.0851 & 5.905 & $0.1433(0.7568)$ & -45.8863 \\
Gamma & 14.9999 & 1.3698 & 4.507 & $0.2573(0.0905)$ & -50.8466 \\
Log-normal & 0.2395 & 0.0936 & 7.642 & $0.2555(0.1132)$ & -51.8826 \\
Gen. Exp. & 38.0767 & 0.3758 & 7.062 & $0.2648(0.0914)$ & -53.4365 \\
Normal & 10.9513 & 0.4683 & 4.479 & $0.1960(0.3667)$ & -47.9054 \\
& (mean) & (variance) & & & \\
\hline
\end{tabular}

Table 6: Estimated parameters, K-S distances, log-likelihood values and the fitted chi-square values for different distribution functions of Dataset 2.

\begin{tabular}{|c|c|c|c|c|c|}
\hline Distribution & Shape & Scale & Chi-square & Kolmogorov & Log-likelihood \\
& & & & & \\
Weibull & 2.8045 & 0.1030 & 5.007 & $0.1873(0.1971)$ & -85.9770 \\
Gamma & 6.3305 & 0.7358 & 2.223 & $0.1634(0.3137)$ & -85.5922 \\
Log-normal & 0.4087 & 0.1260 & 1.040 & $0.1608(0.3607)$ & -85.6416 \\
Gen. Exp. & 10.2548 & 0.3432 & 1.188 & $0.1617(0.3538)$ & -85.6909 \\
Normal & 8.6032 & 0.2956 & 6.528 & $0.1989(0.1467)$ & -85.6209 \\
& (mean) & (variance) & & & \\
\hline
\end{tabular}


Table 7: Estimated parameters, K-S distances, log-likelihood values and the fitted chi-square values for different distribution functions of Dataset 3.

\begin{tabular}{|c|c|c|c|c|c|}
\hline Distribution & Shape & Scale & Chi-square & Kolmogorov & Log-likelihood \\
& & & & & \\
Weibull & 4.0414 & 0.1472 & 6.0954 & $0.2195(0.3509)$ & -34.9123 \\
Gamma & 13.3289 & 2.1696 & 3.8959 & $0.1933(0.4676)$ & -34.4537 \\
Log-normal & 0.1691 & 0.2760 & 3.2291 & $0.1929(0.5145)$ & -34.3606 \\
Gen. Exp. & 45.0044 & 0.7167 & 2.7140 & $0.1944(0.5041)$ & -34.3361 \\
Normal & 6.1441 & 0.5916 & 5.6046 & $0.2115(0.3962)$ & -34.9905 \\
& (mean) & (variance) & & & \\
\hline
\end{tabular}

Table 8: Estimated parameters, K-S distances, log-likelihood values and the fitted chi-square values for different distribution functions of Dataset 4.

\begin{tabular}{|c|c|c|c|c|c|}
\hline Distribution & Shape & Scale & Chi-square & Kolmogorov & Log-likelihood \\
& & & & & \\
Weibull & 2.7520 & 0.5318 & 3.9230 & $0.1389(0.4234)$ & -40.6037 \\
Gamma & 4.7699 & 2.8517 & 6.6101 & $0.1508(0.3008)$ & -43.1471 \\
Log-normal & 0.4056 & 0.5118 & 10.3127 & $0.1640(0.2324)$ & -46.1947 \\
Gen. Exp. & 5.4811 & 1.3925 & 8.7322 & $0.1511(0.3200)$ & -44.5283 \\
Normal & 1.6723 & 0.4537 & 4.1914 & $0.1324(0.4842)$ & -40.9528 \\
& (mean) & (variance) & & & \\
\hline
\end{tabular}


Table 9: Actual and expected number of observation at different intervals for different distribution functions of Dataset 1.

\begin{tabular}{|c|c|c|c|c|c|c|}
\hline Interval & Observation & Weibull & Gamma & Log-normal & Gen. Exp. & Normal \\
$<6$ & 1 & 0.18 & 0.47 & 0.18 & 0.32 & 0.22 \\
$6-9$ & 3 & 5.04 & 5.22 & 2.89 & 5.58 & 3.74 \\
$9-11$ & 5 & 6.85 & 6.22 & 7.16 & 5.99 & 7.23 \\
$11-13$ & 9 & 5.39 & 5.23 & 8.94 & 4.58 & 7.09 \\
$>13$ & 4 & 4.54 & 4.86 & 2.83 & 5.52 & 3.71 \\
\hline
\end{tabular}

Table 10: Actual and expected number of observation at different intervals for different distribution functions of Dataset 2.

\begin{tabular}{|c|c|c|c|c|c|c|}
\hline Interval & Observation & Weibull & Gamma & Log-normal & Gen. Exp. & Normal \\
& & & & & & \\
$<4$ & 1 & 2.63 & 1.92 & 1.55 & 1.65 & 2.86 \\
$4-6$ & 7 & 4.90 & 5.87 & 6.61 & 6.49 & 4.42 \\
$6-8$ & 10 & 7.00 & 8.09 & 8.61 & 8.57 & 6.88 \\
$8-13$ & 11 & 15.03 & 13.59 & 12.49 & 12.59 & 15.64 \\
$>13$ & 4 & 3.42 & 3.53 & 3.74 & 3.70 & 3.20 \\
\hline
\end{tabular}


Table 11: Actual and expected number of observation at different intervals for different distribution functions of Dataset 3.

\begin{tabular}{|c|c|c|c|c|c|c|}
\hline Interval & Observation & Weibull & Gamma & Log-normal & Gen. Exp. & Normal \\
$<400$ & 1 & 1.99 & 1.54 & 1.41 & 1.29 & 1.81 \\
$401-600$ & 10 & 6.18 & 7.50 & 7.96 & 8.44 & 6.55 \\
$601-800$ & 3 & 7.23 & 6.51 & 6.16 & 5.82 & 7.16 \\
$>801$ & 4 & 2.60 & 2.45 & 2.46 & 2.44 & 2.45 \\
\hline
\end{tabular}

Table 12: Actual and expected number of observation at different intervals for different distribution functions of Dataset 4.

\begin{tabular}{|c|c|c|c|c|c|c|}
\hline Interval & Observation & Weibull & Gamma & Log-normal & Gen. Exp. & Normal \\
& & & & & & \\
$<0.75$ & 3 & 3.06 & 3.32 & 3.51 & 3.17 & 3.42 \\
$0.75-1.25$ & 10 & 8.03 & 9.73 & 10.92 & 10.19 & 7.20 \\
$1.25-1.75$ & 6 & 11.29 & 10.93 & 10.30 & 10.32 & 11.22 \\
$1.75-2.25$ & 12 & 9.84 & 7.89 & 6.70 & 7.11 & 10.34 \\
$2.25-2.75$ & 9 & 5.45 & 4.54 & 3.84 & 4.13 & 5.63 \\
$>2.75$ & 2 & 2.32 & 3.67 & 4.73 & 4.53 & 2.19 \\
\hline
\end{tabular}




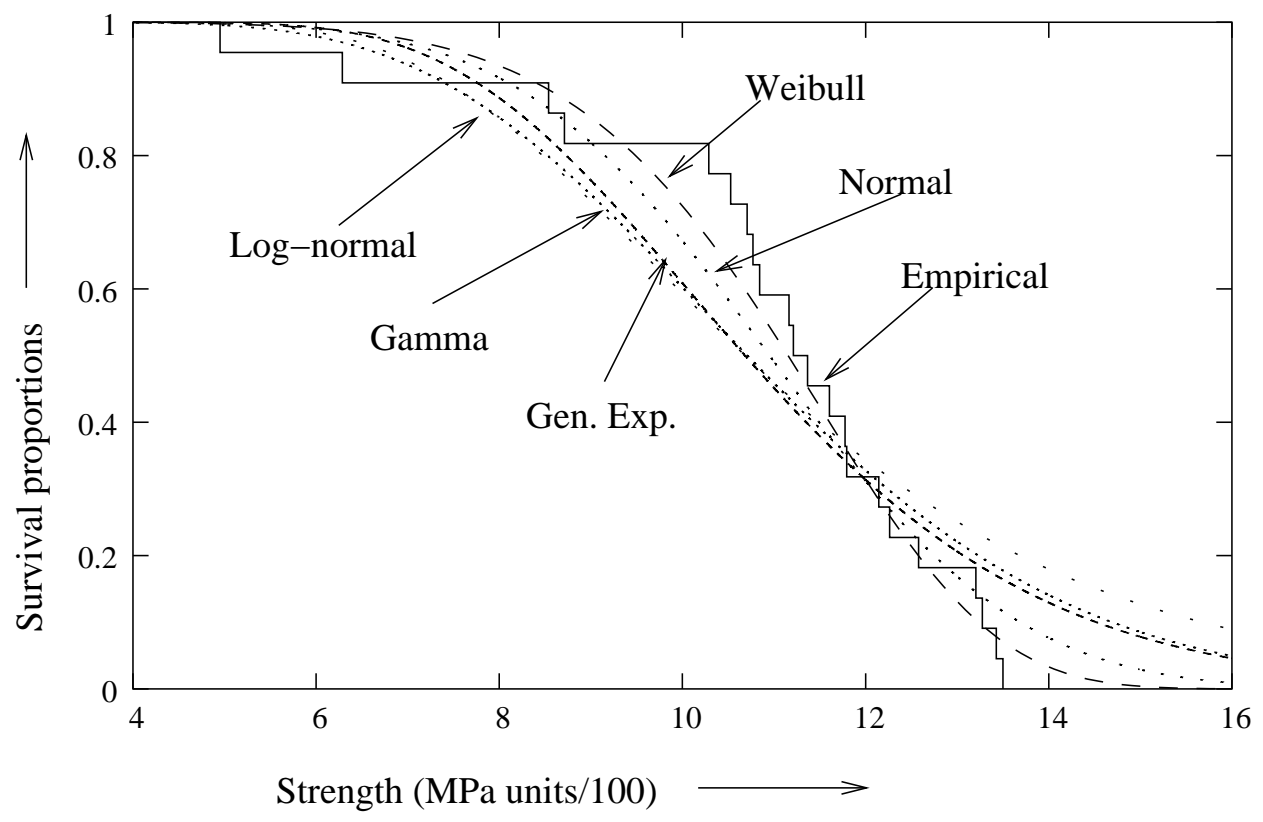

Figure 1: Empirical survival function (bold line) and the fitted survival functions (dotted lines) for Dataset $1\left(\mathrm{ZrO}_{2}-\mathrm{TiB}_{2}\right.$ composite).

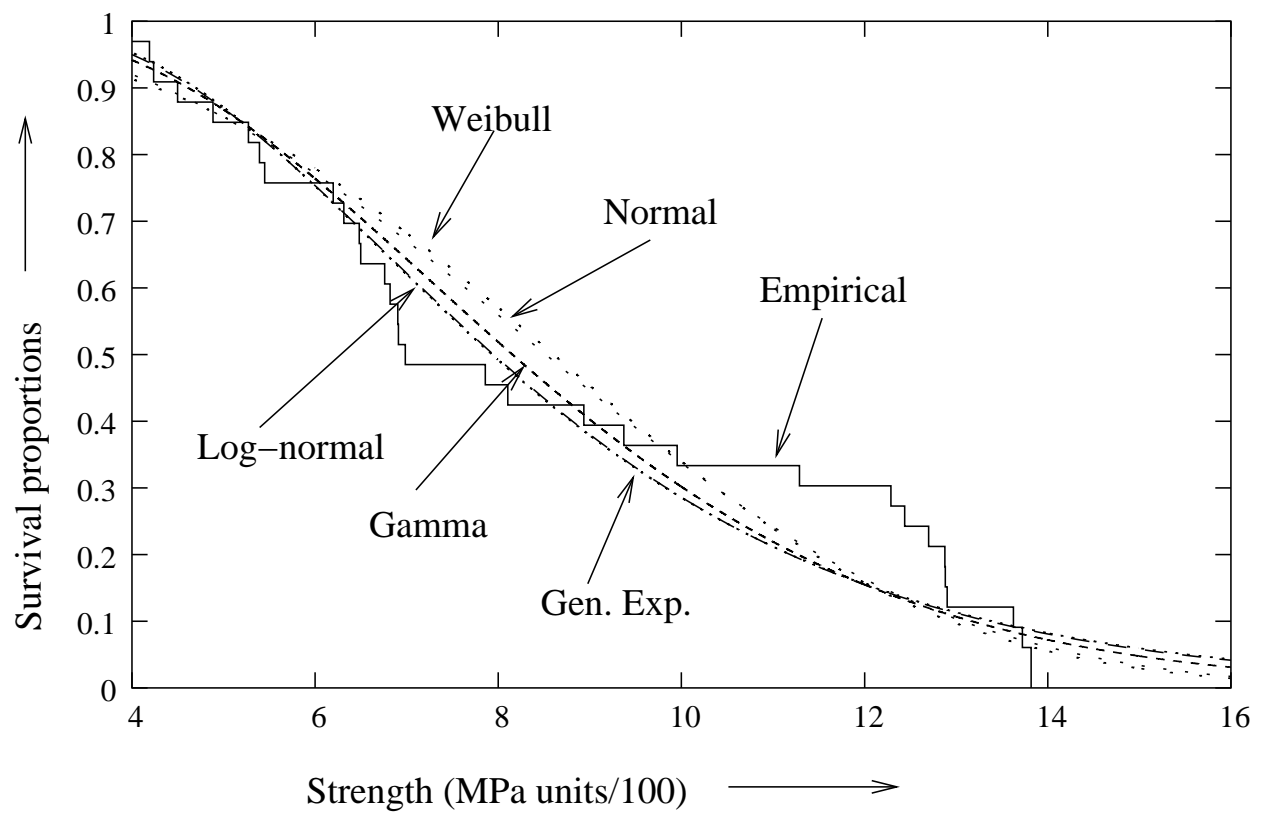

Figure 2: Empirical survival function (bold line) and the fitted survival functions (dotted lines) for Dataset $2\left(\mathrm{ZrO}_{2}\right.$ ceramic). 


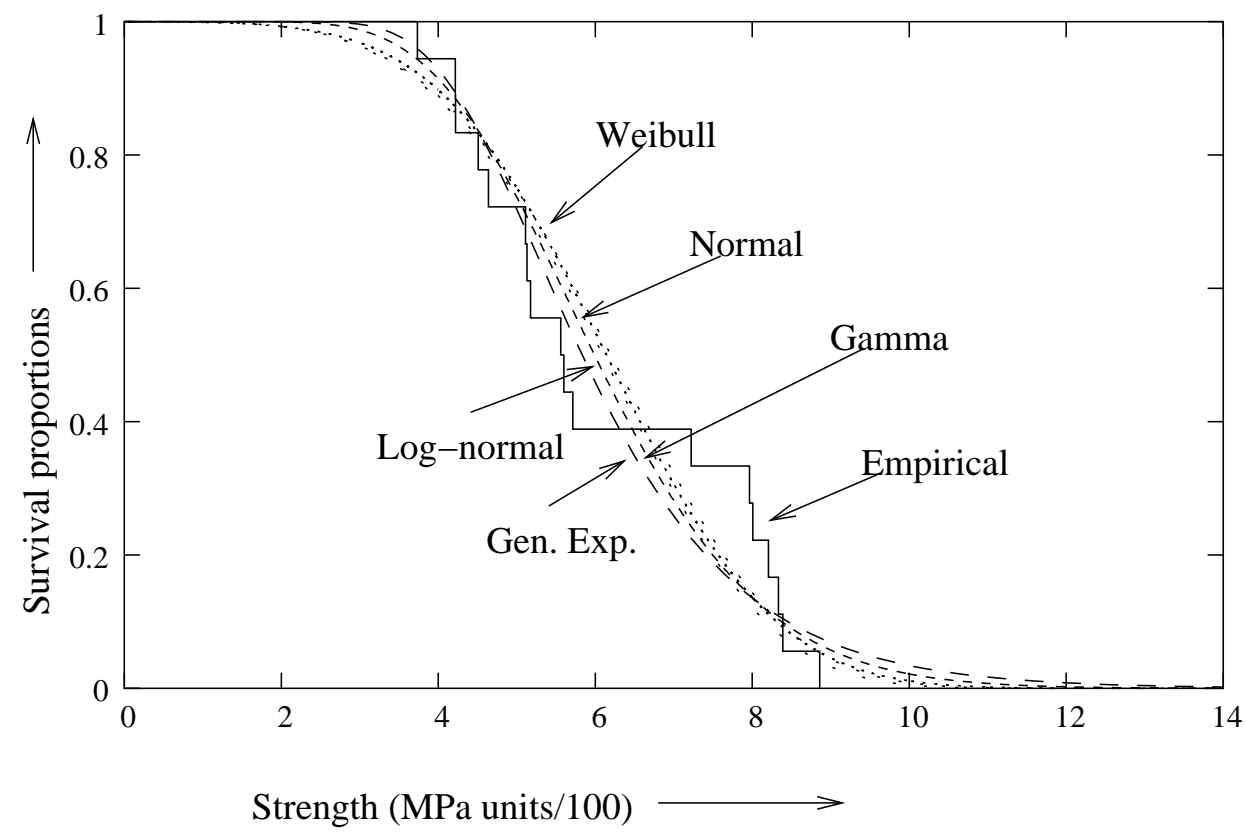

Figure 3: Empirical survival function (bold line) and the fitted survival functions (dotted lines) for Dataset $3\left(\mathrm{Si}_{3} \mathrm{~N}_{4}\right.$ ceramic).

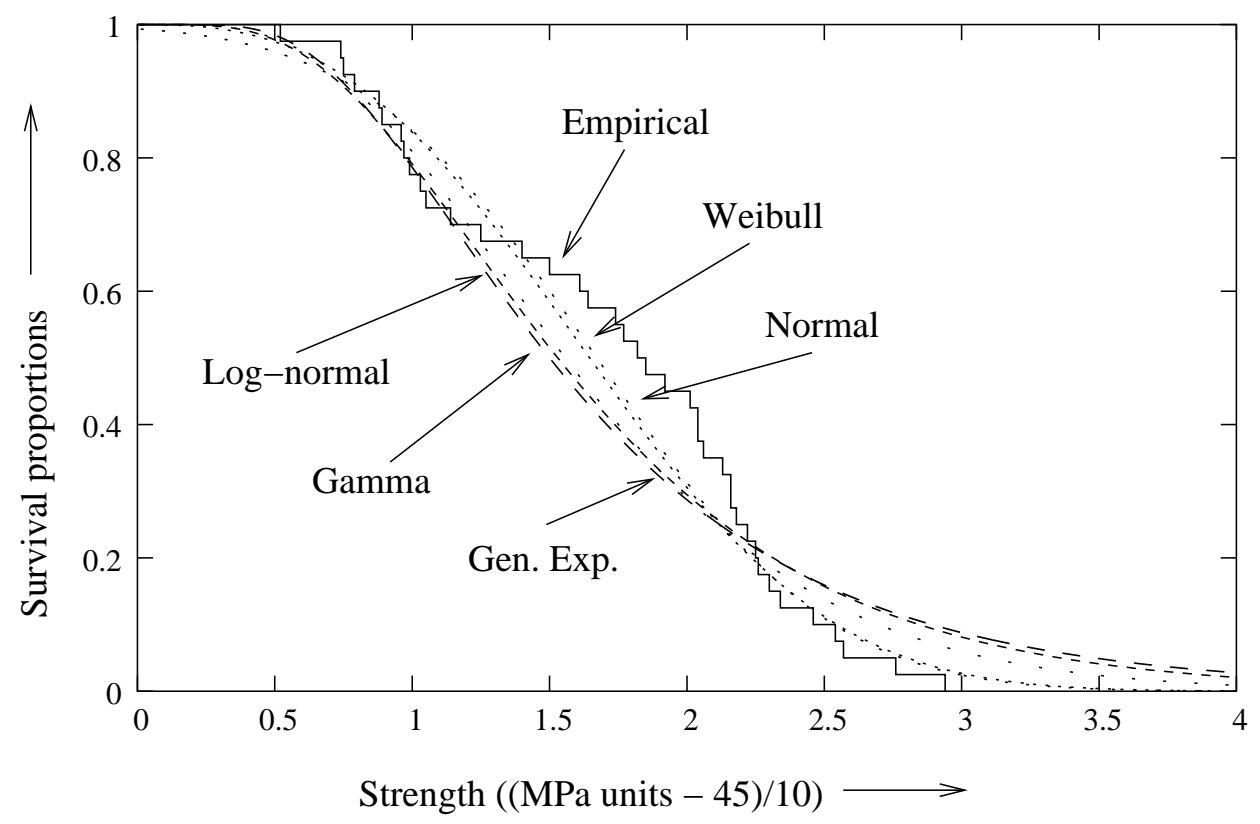

Figure 4: Empirical survival function (bold line) and the fitted survival functions (dotted lines) for Dataset 4 (glass). 